Rhode Island College

Digital Commons @ RIC

$4-28-2021$

\title{
The Effects of Using Ultrasound Guidance Versus Traditional Method for Peripheral Intravenous Placement
}

Jennifer Kennedy

Follow this and additional works at: https://digitalcommons.ric.edu/etd

Part of the Nursing Commons

\section{Recommended Citation}

Kennedy, Jennifer, "The Effects of Using Ultrasound Guidance Versus Traditional Method for Peripheral Intravenous Placement" (2021). Master's Theses, Dissertations, Graduate Research and Major Papers Overview. 382.

https://digitalcommons.ric.edu/etd/382

This Major Paper is brought to you for free and open access by the Master's Theses, Dissertations, Graduate Research and Major Papers at Digital Commons @ RIC. It has been accepted for inclusion in Master's Theses, Dissertations, Graduate Research and Major Papers Overview by an authorized administrator of Digital Commons @ RIC. For more information, please contact digitalcommons@ric.edu. 


\section{THE EFFECTS OF USING}

ULTRASOUND GUIDANCE VERSUS TRADITIONAL METHOD FOR PERIPHERAL INTRAVENOUS PLACEMENT

by

\section{Jennifer Kennedy RN BSN CEN}

A Major Paper Submitted in Partial Fulfillment of the Requirements for the Degree of

Master of Science in Nursing

in

The School of Nursing

Rhode Island College 


\begin{abstract}
Roughly 230 million peripheral intravenous (PIV) catheters are placed in the United States each year (Helm et al., 2015). The establishment of a PIV access may be lifesaving in times when emergent medications or procedures are critical for survival. The literature suggests the use of ultrasound guidance (USG) may decrease the attempts, decrease the length of time to obtain PIV access and improve patient satisfaction. The primary purpose of this study was to compare the longevity of ultrasound guidance PIVs versus the traditional method within the population of patients 18 years and older. A secondary outcome was to determine the most frequently used gauge needle, commonly used location of PIV and infiltration rate. The research was guided by the American Association of College of Nursing's (AACN) Synergy Model as it best provided insight into improving patient outcomes by aligning patient needs and nurse competencies. A retrospective chart review was conducted with a total of 200 charts reviewed. Results concluded as follows: the traditional method was found to be superior in regards to longevity and was also found to have a lower rate of infiltrations. There were a number of limitations to this study such as the lack of information on the training of the health care provider placing the PIV, including the use of the Vascular Access Team or USG training. The number of attempts to obtain PIV access was not available for data analysis. Further research is needed with a specific focus on the extended dwell product along with the additional data collection of factors which may have affected the USG group.
\end{abstract}




\section{Acknowledgements}

To Debra Servello, DNP, APRN-ACNP-BC: Thank you for your positivity and encouragement.

To Jennifer Fearon-Lynch, PhD, RN: Thank you for making me a better writer.

To Julia Twining, MSN, APRN: If it wasn't for your help I wouldn't have completed this

$$
\text { paper. }
$$

To my Mom, thank you for pushing me to apply to the AGACNP and for the countless hours you spent babysitting.

To my husband and daughter. There are no words I can use to express my gratitude and love, everything I do is for you, better together. 


\section{Table of Contents}

Background/Statement of the Problem......................................

Literature Review..........................................................4

Theoretical Framework.......................................................... 11

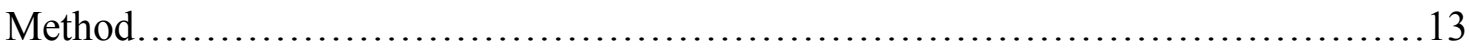

Results................................................................ 16

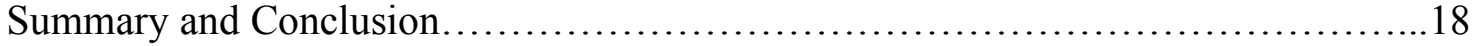

Recommendations and Implications for Advanced Nursing Practice.................22

References............................................................... 24 
The Effects of Using Ultrasound Guidance Versus Traditional Method for Peripheral Intravenous Placement

\section{Background/Statement of the Problem}

Roughly 230 million peripheral intravenous (PIV) catheters are placed in the United States each year (Helm et al., 2015). Establishment of a PIV access may be lifesaving in times when emergent medications or procedures are critical for survival. Therefore, it is pivotal that the registered nurse $(\mathrm{RN})$ not only has the skill set but the resources to fulfill the patients' needs. The level of resources available impacts the level of support nurses need to provide for the patient; the more resources available the greater the potential for a positive outcome (Kaplow \& Reed, 2008).

Obtaining PIV access is an acquired and learned skill in which RNs may develop at varying levels of competencies. It is important that the RN utilizes critical thinking when assessing peripheral access and obtains the nursing skills acquired through evidence-based guidelines to establish patient-centered care (Kaplow \& Reed, 2008). A PIV failure causes an enormous cascade of events including anxiety and stress for both the patient and staff, potential PIV complications, decreased patient satisfaction, additional time and supplies spent on the task and having to potentially treat complications (Helm et al., 2015). Delays in obtaining PIV access may delay diagnosis and treatment for the patient and has been linked to increased incidences of morbidity and mortality (Bridey et al., 2018). Conditions associated with difficult PIV access include, but are not limited to, drug use, obesity, chronic illness, a restricted extremity, and hypovolemia (Fields et al., 2014). Common complications such as infiltration, extravasation, phlebitis, and catheter occlusion account for almost fifty complications per 
day in a 160-bed hospital (Helm et al., 2015). The average time needed for obtaining PIV access ranges from 2.5-16 minutes, with difficult PIV access requiring as much as 30 minutes (Leidel et al., 2009). Significant delays in care were reported when PIV access required physician intervention with times to successful placement ranging from 22-57 minutes (Witting, 2012).

Approaches to obtaining PIV access include the conventional/traditional method of visualization and palpation of the vein. Improper establishment of PIV placement via the traditional/conventional method can lead to infection from the PIV site, phlebitis, infiltration, extravasation, hematoma, thrombophlebitis, or intra-arterial injection from accessing an artery instead of a vein; causing arterial spasm and eventual loss of a limb (Helm et al., 2015). At times, patients with difficult venous access may endure multiple PIV attempts, which could lead to the placement of a central venous catheter if access is not obtained. Central venous catheter (CVC) placement can be associated with increased risk for infection and procedural complications. The Centers for Disease Control and Prevention [CDC] (2011) reports central line associated blood stream infections (CLABSIs) result in thousands of deaths each year and billions of dollars in added costs to the United States. Some of the literature suggests that the use of ultrasound guidance (USG) may decrease the number of attempts, decrease the length of time to obtain PIV access, and improve patient satisfaction (Loon et al., 2018; Shokoohi et al., 2013).

The primary purpose of this study was to compare the longevity of ultrasound guidance PIVs versus the traditional method within the population of patients 18 years and older. The following research question guided the study: In patients requiring peripheral access, is the use of ultrasound guidance shown to have superior longevity 
compared to traditional technique to maintain peripheral IV access? A descriptive retrospective chart review with a two-group design was completed to evaluate the USG PIV compared to traditional insertion. The secondary outcome was to collect data on the most frequent gauge needle utilized and location of PIV placement. 


\section{Literature Review}

For this review, a wide-spread database within the past 10 years was searched including CINAHL, Pub Med, Up-to-date, and Google Scholar. The literature search was limited to the English language only using the key terms "peripheral IV access and ultrasound," "difficult PIV access," and "ultrasound guided peripheral intravenous insertion."

\section{Cannulation}

The term intravenous cannulation refers to a technique in which a cannula is placed inside a vein to provide venous access (Medscape, 2019). A successful IV placement is achieved when the patient is able to complete the task or test that the IV was needed for and there are no signs or symptoms indicating infiltration including pain, redness, swelling, or leaking from the catheter site. A failed attempt is defined as an inability to complete the test/task that the IV was needed for along with pain, redness, and swelling to the site and an inability to flush the IV and or leaking from the catheter site (Bauman et al., 2010). Successful cannulation using USG is defined as withdrawal of $5 \mathrm{~mL}$ of non-pulsating blood or infusion of $5 \mathrm{~mL}$ of solution without extravasation using the USG method. In contrast, a failed attempt with USG insertion is defined as three unsuccessful ultrasound guided attempts. Whereas a failed attempt with the traditional method is defined as an occurrence of infiltration during initial infusion, inability to withdraw blood, or requiring assistance from a health care provider for PIV placement (Bauman et al., 2010). 


\section{Difficult Peripheral Intravenous Access}

Patients with difficult PIV access can be characterized as having non-visible and non-palpable veins and or have the following risk factors: history of difficult PIV access, IV drug use, chemotherapy treatment, obesity, having a restricted extremity, dehydration, or chronic medical conditions (Sou et al., 2017). The following studies looked specifically at patients labeled as having difficult PIV access and the effects of using USG when compared to the traditional approach.

In a systematic review using meta-analysis (Loon et al., 2018), investigators compare USG with a traditional approach of visualization and vein palpation. The authors had done so based on existing research suggesting the use of several different methods to aid in the achievement of successful PIV cannulation including USG. The meta-analysis followed the guidelines of the preferred reporting items for systematic reviews and meta-analysis. Eight studies were selected in the final analysis with a total of 1640 patients. The authors found an improved success rate for PIV access when using USG compared to the traditional method. They also concluded that the use of ultrasound reduced the number of punctures, improved speed of procedure, and increased patient satisfaction. The rate of complications with USG was no different when compared to the traditional method (Loon et al., 2018). The authors recommended further research to develop a risk measurement scale to classify potential need for ultrasound use for patients and to further demonstrate the efficiency and efficacy of USG (Loon et al., 2018).

The placement of PIV catheters can be performed by varying levels of providers including residents, doctors, nurses, and specially trained emergency department (ED) technicians. The following study looked specifically at ED technicians. Bauman et al. 
(2010) in their experimental study with a sample of 75 patients, also recognized the difficulty of obtaining PIV access for some patients and its consequences. The study utilized ED technicians with a minimum of one-year experience trained to use USG during the study; instead of physician trained as compared to previous studies. The authors identified the use of USG PIV placement to be an effective intervention for the problem of difficult PIV access despite similar success rate for both the USG and the traditional method. The ED technicians were trained only to use the upper arm basilic and brachial veins, limiting vein selection and size of needle length and gauge when using the USG approach. In contrast, the authors did not limit the vein selection or type of needle to be used for the traditional technique.

Bridey et al. (2018) identified a common problem in their ICU as it relates to difficulty in placing PIV catheters for the purpose of removing central venous catheters when appropriate. There was a total of 114 patients enrolled in the study, 57 in each group (USG and the traditional method). One case was lost in the USG and one patient did not receive intervention due to clinical deterioration. The results showed that both the number of attempts and the catheter's lifespan were similar in each of the groups. Other findings revealed extravasation occurred more frequently in the ultrasound method, patient satisfaction showed similar results in each group, and nursing satisfaction was improved in the ultrasound method compared to the traditional method. Based on the findings, it was concluded that the utilization of the USG for peripheral intravenous access did not reduce the number of attempts compared to the traditional approach.

Aponte et al. (2009), investigated the comparison between the success rate and time it took to place a PIV catheter in either the hand or forearm veins of adults with 
difficult peripheral access via the traditional and USG method. This quantitative study consisted of thirty-five adults with a history or suspicion of difficult PIV access in an operating room. The results revealed there were no significant differences between the groups, with the use of USG to be as effective as the traditional method. The authors suggested that further investigation should assess the effectiveness of the USG technique after a failed attempt by the traditional method. The authors did not identify the use of a framework in their research. In addition, the authors stated that when patients selfreported a history of difficult PIV cannulation, they were not truly difficult.

\section{Decline in Central Venous Catheter Placement}

A Central Venous Catheter (CVC) is a catheter placed into a large vein located in the neck. When warranted, the placement of a CVC may be lifesaving for the critically ill patient who needs central venous pressure monitoring and administration of certain medications. However, several complications may result during and after a CVC is placed including pneumothorax, bloodstream infections, thrombosis, and hemorrhage (CDC, 2011). The following study looked to lower the rate of CVC placements in noncritical or non-emergent cases with the utilization of USG for PIV placement.

Shokoohi et al. (2013), hypothesized that the utilization of USG may reduce the need for $\mathrm{CVC}$ placement in non-critically ill patients in the emergency department. The authors identified the importance of obtaining PIV access in emergency departments where CVC placement is a common practice when access was not gained. The design utilized was a retrospective cohort study, using a time-series analysis of the monthly rate of CVC during the study period. The research was conducted over a six-year period, with a total of 401,532 patients who were treated; 1,583 of whom received a CVC. The annual 
patient admission rate increased in the emergency department; however, the overall CVC placement rate decreased by a total of $80 \%$ at the end of the study. The non-critically ill patients specifically had a significant reduction in CVC placement and by the end of the study in 2011, there was no CVC placement in emergency department patients who were discharged home from the department. The authors reported that the study did provide direct evidence that the USG program caused the reduction in the central venous catheter rate and recommended rigorous research specific to that area of interest.

There were several limitations identified in the above study, however. Most importantly, the researchers did not compare the traditional method with the use of USG. Therefore, the authors could not state if the cause of the decrease in CVC was in relation to the use of USG. Furthermore, there was no record of the number of failed attempts for either the traditional method or USG. The authors also stated that the total number of CVCs placed may have been underestimated among patients for whom the procedure was not properly documented. There was also no mention of needle size or required location for USG instructions for the user. Teaching and training levels differed for the residents as well. A strength of the study was the large sample size and extensive training provided for the resident group.

\section{Patient Satisfaction}

Patient satisfaction is an integral part of healthcare which can be a predictor of quality care (Pandurangadu et al., 2016). Prakash states that "patient satisfaction affects clinical outcomes, patient retention, and medical malpractice claims. It affects the timely, efficient, and patient-centered delivery of quality health care" (2010, p. 151). Nursing interventions (e.g., difficult PIV cannulation) can impact patients' physiological, 
psychological and emotional stability (Kaplow \& Reed, 2008), thus impacting patients' overall satisfaction scores. Hospital reimbursement is also affected by the Centers for Medicare and Medicaid Services (CMS) that rate patient satisfaction and their hospital experience on the Hospital Consumer Assessment of Healthcare Providers and Systems. Patients with difficult peripheral access are at risk for an increased number of PIV attempts, due to obesity, IV drug use, and end stage renal disease (Pandurangadu et al., 2016). Prevalence of conditions associated with difficult PIV access are predicted to rise which may increase the number of patients with difficult PIV access. The following study assessed the impact of patient satisfaction when nurse performed USG peripheral IV placement was implemented.

Pandurangadu et al., 2016, performed a randomized, prospective controlled study which included the training of USG peripheral IV placement of emergency department nurses. The study was conducted at a level 1 trauma hospital with an annual ED census greater than 125,000 and nurses had no previous formal training in ultrasound-guided peripheral IV placement. Emergency Department nurses having at least two years of good standing experience were notified of the study and volunteered to participate. A total of 124 patients were enrolled and randomly assigned to the study. Pandurangadu et al. (2016) measured median patient satisfaction using a 10-point Likert scale and found that satisfaction was enhanced when the USG PIV method was used (10/10) compared to the traditional technique for patients with difficult peripheral access (8/10). Additionally, authors recommended the training of ED nurses for patients with difficult peripheral access to enhance the patient care experience. 
In summary, PIV access can be a daunting task for the provider and the patient. Delays in obtaining access have been shown to increase the risk of morbidity and mortality (Bridey et al., 2018). The use of USG has been studied and evaluated for its efficiency and efficacy at times when PIV access is not obtainable. The literature review did not conclusively determine whether PIV access would be more attainable or sustainable with the aid of USG compared to the traditional method. However, several authors recommended proper USG training as it has the potential to have efficacy in patients with poor venous access (Bauman et al., 2010; Bridley et al., 2018; Pandurangadu et al., 2016). Based on the result of the literature review, further research is suggested with a focus on USG training to determine if the use of ultrasound will be more effective compared to other techniques. Despite the literature having different outcomes, authors in this literature review had similar gaps in their studies. One of the gaps is the lack of clarity in the term "difficult access," which could potentially cause differing patient populations to be analyzed. For example, not one of the studies identified if the patient was restricted to one arm or if that was a deciding factor in why a patient was labeled as having difficult access. Another common feature was that the needle selection and location of the PIV catheter in each study all varied. In addition, researchers did not identify if a limitation of depth was set for the use of USG or the view of the probe. There were also varying levels of training and expertise of the nurses. Furthermore, authors from the literature review did not use a theoretical framework to help guide their research. Theoretical frameworks help establish structure and guide the research to investigate the identified problem (McEwen \& Wills, 2018). Utilizing a theory helps reduce biases that may sway the researcher's interpretation of results. 


\section{Theoretical Framework}

\section{AACN Synergy Model for Patient Care}

The American Association of College of Nursing's (AACN) Synergy Model for patient care was selected to help guide the study of research. The AACN Synergy Model is a conceptual framework that provides support of patient care needs and nurse competencies. The major concept of the model is to match nurse competencies with patients' needs to optimize patient outcomes (Curley, 1998). The AACN's Synergy Model is recognized to be a professional practice model, a foundation for nursing school curricula, and a model for professional advancement such as nursing certification. The model was developed by AACN for critical care patients by nurses for practice use. Despite its scope of origin, it has been adapted to other disciplines and used in a range of different patient populations and settings (McEwen \& Wills, 2018). For example, the Synergy Model has informed the development and interventional guide for "Rapid Response Teams" in order to provide medical assistance for admitted patients experiencing a sudden change in their condition (McEwen \& Wills, 2018).

There are eight patient needs identified in the model: complexity, resource availability, participation in decision-making, vulnerability and predictability, participation in care, resiliency, and stability. Along with the identified patient needs are the nursing competencies which include clinical judgment, clinical inquiry, facilitation of learning along with advocacy and moral agency, caring practices, collaboration, systems thinking and response to diversity. The model describes three levels of outcomes which correlates with the patient, nurse and the health care system. Patient outcomes include functional and behavioral changes, trust, satisfaction, comfort and quality of life. Nursing 
outcomes encompass physiological changes, presence or absence of complications and the extent to which care objectives were obtained. The central idea of the model is that the patient's need drives the nurse competencies required for patient care (Kaplow \& Reed, 2008).

Although concepts of the model were originally developed for critical care patients and nurses, it can be applied to many areas of research. Hence, the synergy model has been selected to guide the PICO question for this study: In patients requiring peripheral access, is the use of ultrasound guidance shown to have superior longevity compared to traditional technique to maintain peripheral IV access? The model can be useful in guiding the research because it specifically seeks to improve patient outcomes by aligning patient needs and nurse competencies as previously mentioned. Obtaining PIV access can be problematic even for the most experienced nurse. A delay in obtaining access can result in several adverse events including infection, infiltration, placement of central venous catheter, and delay in care. The Synergy Model can support the need for USG training for all nurses who provide PIV access to improve patient outcomes.

The use of the framework for the previously mentioned PICO question will help guide the research by identifying the patient characteristics of those with limited peripheral access and nurse competencies necessary to optimize patient outcomes. With this model as a guide, the study will help provide insight into how licensed independent practitioners can advocate for their patients by considering the use of USG PIV access for those who require PIV access. The methods guiding this study will be presented in the next section. 


\section{Method}

\section{Purpose}

The primary purpose of the study was to compare the longevity of ultrasound guided PIV catheters versus the traditional method for adults 18 years of age and older requiring admission into the hospital. A successful PIV placement was defined as follows: no pain, redness, swelling, induration, or leaking from the catheter site. The primary outcome was to determine if In patients requiring peripheral access, is the use of ultrasound guidance shown to have superior longevity compared to traditional technique to maintain peripheral IV access? Success was defined as follows: without pain, redness, swelling, induration or leaking from the catheter site. The secondary outcome was to determine most frequently used gauge needle, location of PIV and infiltration rate.

\section{Design}

The study was a descriptive retrospective chart review with a two-group design: ultrasound guided PIV as compared to traditional insertion.

\section{Study Setting and Population}

The data collection was conducted at Miriam Hospital, a 247-bed acute care teaching hospital located in Providence, Rhode Island. Inclusion criteria included male and female patients 18 years of age and older who received care as inpatients with a PIV catheter ordered. Exclusion criteria included patients who did not have a PIV catheter ordered. 


\section{Procedure}

Approval was obtained from the Chief Nursing Officer. Permission was granted by the manager of the Intravenous Therapy (IVT) Department at the research site to conduct the study. The Information Systems (IS) LifeChart team was contacted via a report request to extract data through the LifeChart (Epic) Clarity Database for the detailed data points based on the study population. Data was collected on patients admitted between December 2019 through February 2020

A total of 200 charts were reviewed by April 2021 with IRB approval. The medical records of potential subjects were reviewed by the student for inclusion and exclusion criteria. The records that meet inclusion criteria were reviewed by the IS LifeChart team further to extract the identified data. No patient identifiers were collected. Data was reviewed with the IS LifeChart team during regular office hours and entered in an Excel spreadsheet. Confidentiality of the data was maintained throughout the course of the study. Data retrieved were kept on an encrypted flash drive and in a locked box stored in the IV therapy department. All data were destroyed upon the completion of the study's evaluation.

\section{Measurement}

A worksheet (see Table 1) developed by the student was used to gather data. Only data meeting the inclusion criteria without identifying information were collected by the IS LifeChart team at research site. The collection tool measured the direct comparison of longevity between the two methods of PIV placements. Data on location of PIV, if the PIV was removed, reason for removal including the rate of infiltration was collected. Data was also collected on needle gauge and location of PIV. 


\section{Overview of the IRB Process/ Ethical Considerations}

The student obtained IRB approvals from the clinical site and the Rhode Island College IRB during September and November 2020. Data request began immediately following IRB approval. Data analysis was completed by April $16^{\text {th }} 2021$. Patient confidentiality was maintained by utilizing de-identified data.

\section{Analysis}

Collected data were entered into Microsoft Excel for analysis. Basic descriptive statistics was utilized along with direct comparison of longevity between the two methods of PIV placements. The rate of PIV infiltration for each group was also analyzed by percentage. Descriptive statistics such as central tendencies of mean, median and mode were used to assist with analysis. 


\section{Results}

All 200 charts reviewed met the inclusion criteria. Reviewed charts included 100 that received USG for PIV placement, and 100 in the traditional method group. PIVs were removed accidentally in $0.04 \%$ of the traditional cases. Removals were indicated for infiltration, tenderness, leaking or occlusion, there were no documented removals due to infiltration, tenderness, leaking or occlusion in the traditional cases. The average number of hours the PIV lasted in this group was 42.33 (1.76 days). The longest duration was 141 hours (5.87 days) and shortest duration was 3 hours. The average length of stay for the traditional group was 1.85 days. The most frequently needle gauze used was size 20 $(0.76 \%)$ with $0.55 \%$ placed in the antecubital site. Eighteen-gauge needles lasted an average of 1.26 days with 2.25 being the longest amount of days maintained and 0.58 days being the least. The 22-gauge needles lasted an average of 2.48 days with 9.5 days being the longest and 0.58 days the shortest duration. The 20- gauge needle group was found to have an average of 1.73 days lasted, 5.87 days being the longest and 0.16 the shortest in duration with the traditional group. The most common $(0.76 \%) 20$-gauge needle lasted an average of 1.73 days, 5.87 days being the longest and 0.16 the shortest in duration with the traditional group. PIVs were removed due to infiltration $(0.11 \%)$, tenderness $(0.05 \%)$, leaking $(0.02 \%)$, occlusion or accidental removal $(0.02 \%)$ in the USG group. The average number of hours the PIV lasted in the USG group was 59.67 (2.5 days) with the longest being 10.67 days and shortest less than one hour $(0.0 \%)$ ). The average length of stay in this group was 6.52 days. A chart of comparisons is seen below in Figure 1. For the USG group, $0.86 \%$ used a 20 -gauge needle with $0.45 \%$ chosen the upper arm as the most frequent site for placement. The 22-gauge needles were used in the 
USG with a duration of 1.08 days. Eighteen-gauge needles lasted an average of 1.95

days, with 5.16 days being the longest and 0.41 days the shortest duration.

\section{Figure 1}

Comparison Between Traditional and USG Method

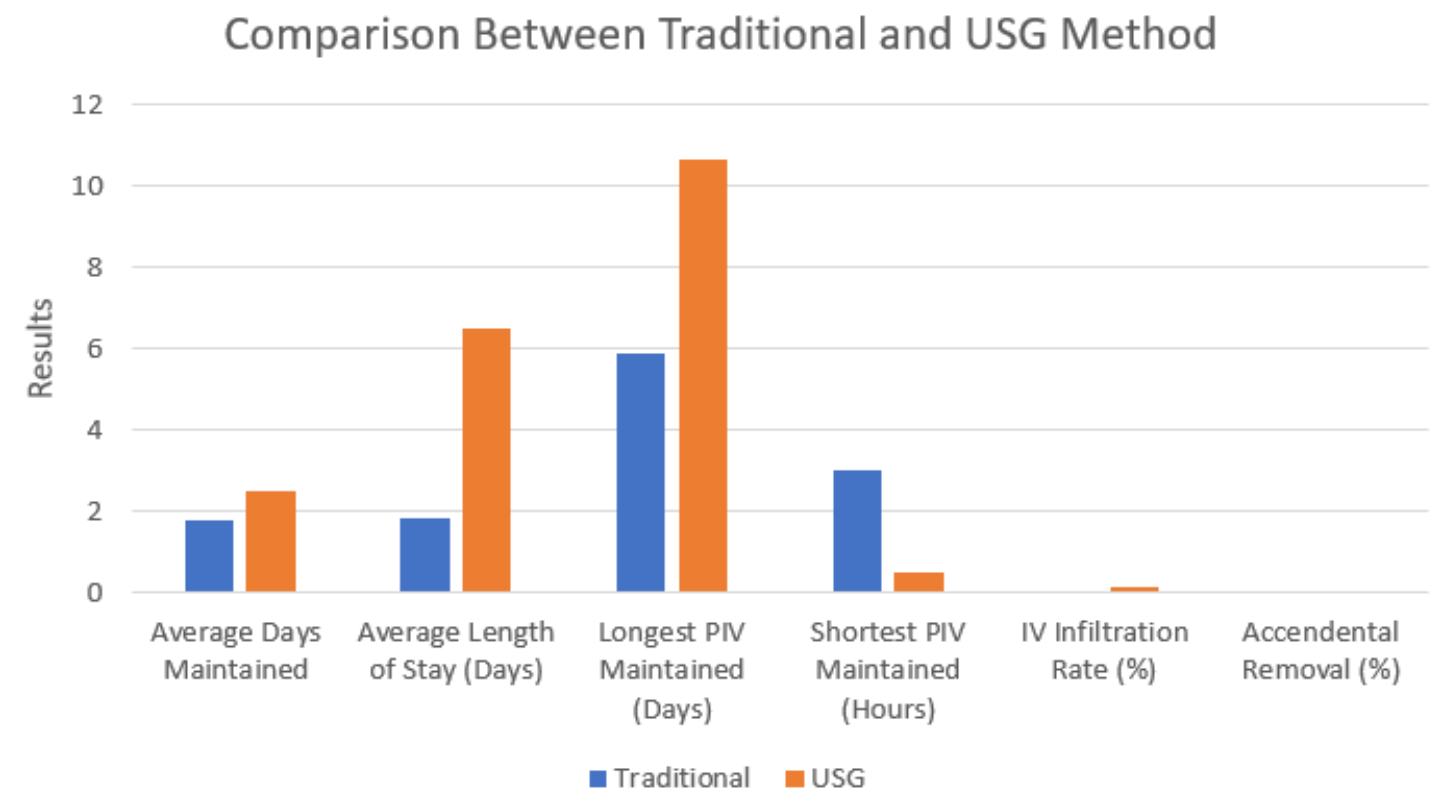




\section{Summary and Conclusions}

A PIV failure causes an enormous cascade of events including anxiety and stress for both the patient and staff, potential PIV complications, decreased patient satisfaction, additional time and supplies spent on the task and having to potentially treat complications (Helm et al., 2015). Delays in obtaining PIV access may delay diagnosis and treatment for the patient and has been linked to increased incidences of morbidity and mortality (Bridey et al., 2018).

In summary, the literature review did not conclusively determine whether PIV access would be more attainable or sustainable with the aid of USG compared to the traditional method. However, several authors recommended proper USG training as it has the potential to have efficacy in patients with poor venous access (Bauman et al., 2010; Bridley et al., 2018; Pandurangadu et al., 2016).

The primary purpose of this study was to compare the longevity of USG PIVs versus the traditional method within the population of patients 18 years and older. The following research question guided the study: In patients requiring peripheral access, is the use of ultrasound guidance shown to have superior longevity compared to traditional technique to maintain peripheral IV access? A secondary outcome was to determine the most often used gauge needle, location of PIV and infiltration rate. The study was guided by the Synergy Model (Curley, 1998). A retrospective chart review was conducted. Two hundred charts meeting the inclusion criteria were randomly selected and analyzed, 100 for each group. The researcher's projected outcome was for the USG to have a superior longevity when compared to the traditional method group. 
The statistics from this retrospective chart review demonstrated the USG group had an average of 2.5 days longevity with an average length of hospital stay (LOS) of 6.52. The most common reason for removal of PIV, other than being discharged, was due to infiltration. The USG group had a longer LOS when compared to the traditional method, this could be related to a complicated hospital course, comorbidities, or presenting illness; however, this data was not collected. The difference between the LOS and average longevity of USG PIV was 4 days.

The traditional method group demonstrated a zero occurrence of infiltrations. The most common reason for removal other than discharge was accidental. The average number of days the PIV sustained were 1.76 with a LOS of 1.85 days. Despite the ultrasound group having a longer number of days sustained, the difference between maintained PIV days to LOS for the USG group was longer; a difference of 4.02 days compared to 0.09 days for the traditional group. The difference between the LOS and average longevity of the traditional PIV was 0.09 days.

The 20-gauge needle was the common gauge needle choice in both groups, with $(0.76 \%)$ in the traditional group and $(0.86 \%)$ in the USG method. The location of PIV placement varied; the antecubital being the most common site for the traditional method and upper arm in the USG group. The Infusion Nurses Society standards of practice suggest when selecting a needle gauge, one should utilize the smallest gauge and length to accommodate and manage the prescribed therapy (Infusion Nursing, 2015).

There were a number of limitations to this study. There was a lack of information on the training of the health care provider placing the PIV, including the use of the Vascular Access Team or USG training. No data was collected on the level of perceived 
or known history of limited PIV access including medical condition(s) and comorbidities. Documentation error may have occurred with charting. The number of attempts to obtain PIV access was not available for data analysis. The use of other USG products was not collected. The timeline of data collected was during the start of a pandemic when maximum personal protective equipment (PPE) were being used, this may have decreased the health care providers ability to visualize and palpate the vein.

Conclusions from the research question are as follows: the traditional method was found to be superior in longevity in relation to the average LOS, including a lower rate of infiltrations with the most common gauge needle used being 20 gauge, in the antecubital. It can be concluded that despite direct visualization of veins with the use of USG, the traditional method PIV can last longer in relation to the patient's hospital course, having fewer complications. These findings may improve patient and nurse satisfaction along with decreasing hospital cost. It is also important to recognize the USG method group had a longer LOS, therefore it can suggest that those requiring increased LOS, the traditional method for PIV placement may be indicated. Despite the USG group having an average 2.5 days that the PIV was maintained, there was a longer LOS, meaning the PIV did not last as long in relation to the patient's hospital stay.

The upper arm was the most common site used for PIV placement in the USG group. Therefore, it can be concluded from these results that the placement of USG PIVs using the anatomical location of the upper arm leads to higher rates of infiltration compared to the traditional method. In a study conducted by Rupp et al. (2016) in patients who had a computerized tomography (CAT) scan with contrast, USG PIVs had a higher risk of extravasation compared to those who received contrast through a standard 
PIV. Further research is needed with a specific focus on the extended dwell product along with the added data collection of factors which may have affected the USG group including acuity of illnesses(s) and comorbidities. Additional research regarding the prevention of PIV catheter associated events such as infiltration is also indicated and clinicians should consider the infiltration risk when weighing the risk and benefits of USG PIV for vascular access. Current literature emphasizes the importance of preserving patients' peripheral veins for access including for future vascular access needs (Carr et al., 2016). With each successive PIV placement attempt, the vein availability decreases. With every attempt to insert a PIV catheter, micro-tears occur in the vessel intima that can lead to infiltration and extravasation. Furthermore, when a catheter is advanced that is not fully seated within the vein lumen, the chances of infiltration and phlebitis increase (Helm et al., 2015).

\section{Plan for Dissemination}

Dissemination of the findings was distributed to hospital staff who are specially trained in USG and to paramedics who have not yet started incorporating USG for PIV placement. The findings were distributed during PIV training classes at the clinical site and published in the Rhode Island College Digital Commons. 


\section{Recommendations and Implications for Advanced Nursing Practice}

The Advanced Practice Nurse (APRN) which includes the Acute Care Adult Nurse Practitioner are pivotal providers in healthcare. The APRN encompasses core competencies of direct clinical practice, expert coaching and guiding, consultation, research, clinical and professional leadership. While keeping the triple aim framework as an objective, the APRN needs to have forward thinking with a consistent strive to improve the experience of care while keeping the cost of care in mind. This study demonstrated the use of USG products had a higher rate of infiltration when compared to traditional method. Prevention of PIV associated events such as infiltrations, should be the preferred approach with PIV placement. Promoting the need for maintaining awareness along with reinforcing provider education on the importance in this area. The APRN can assist with the implementation of a PIV insertion education bundle to increase staff recognition of infiltrations.

As a mentor and educator, the APRN can improve the standard of practice and improve patient experience by reviewing and applying up-to-date evidence. Evidence derived care can then form the basis for policy recommendations which can aid with consistent practice for the nursing team. Nursing practice inconsistencies directly affects patient outcomes. It is important the $\mathrm{RN}$ uses critical thinking when assessing peripheral access and obtains the nursing skills acquired through evidenced-based guidelines to establish patient-centered care (Kaplow \& Reed, 2008). As patient satisfaction has appeared as a key quality indicator, it is now an important determinant for accreditation. While keeping this in mind, the APRN can advocate for the re-implementation of educational programs hospital wide on PIV insertion with a focus on traditional 
technique. In summary, educating all providers involved with PIV placement including the prevention of infiltration, provider education, clinical vigilance by all involved healthcare providers, including stock holder, patients and families creates a shared responsibility with mutual respect. 


\section{References}

Aponte, C., Acosta, S., Rigamonti, D., Austin, P., \& Samolitis, T. (2009). The use of ultrasound for placement of intravenous catheters. American Association of Nurse Anesthetists Journal, 73(3), 212-216. https://doi.org/10.21236/ada428738

Bauman, M., \& Braude, C. (2010). Ultrasound-guidance vs. standard technique in difficult vascular access patients by ED technicians. The American Journal of Emergency Medicine, 27(2), 135-140. https://doi.org/10.1016/j.ajem.2008.02.005

Bridey, C., Thilly, N., Leferre, T., Maire, R., Morel, M., Levy, B., Girer, N., \& Kimmoun, A. (2018). Ultrasound-guided versus landmark approach for peripheral intravenous access by critical care nurses: A randomised controlled Study. British Medical Journal Open, 8(6), 100-122. https://doi.org/10.1136/bmjopen-2017$\underline{020220}$

Carr, P. J., Rippey, J. C. R., Cooke, M. L., Bharat, C., Murray, K., Higgins, N. S., Rickard, C. M. (2016). Development of a clinical prediction rule to improve peripheral intravenous cannula first attempt success in the emergency department and reduce post insertion failure rates: The vascular access decisions in the emergency room (VADER) study protocol. British Medical Journal Open, 6(2), 1-7. https:// doi:10.1136/bmjopen-2015- 009196

Center for Disease Control and Prevention. (2011). Guidelines for the preventions of intravascular catheter-related infections. Retrieved from http://www.cdc.gov/hicpac/BSI/references-BSI-guidelines-2011.html

Curley, M. (1998). Patient-nurse synergy: optimizing patients' outcomes. The American Journal of Critical Care, 7(1), 64-72. https://pubmed.ncbi.nlm.nih.gov/9429685/ 
Fields, M., Piela, N., Au, A., \& Ku, B. (2014). Risk factors associated with difficult venous access in adult ED patients. The American Journal of Emergency Medicine, 32(10), 1179-1182. https://doi.org/10.1016/j.ajem.2014.07.008

Helm, R. E., Klausner, J. D., Klemperer, J. D., Flint, L. M., \& Huang, E. (2015). Accepted but unacceptable: peripheral IV catheter failure. Journal of infusion nursing: the official publication of the Infusion Nurses Society, 38(3), 189-203. https://doi.org/10.1097/nan.0000000000000324

Infusion Nursing. (2015). Infusion therapy resources and references. Retrieved from https://infusionnurse.org/2015/11/23/what-iv-needle-size-should-i-use/

Kaplow, R., \& Reed, K. (2008). The AACN synergy model for patient care: a nursing model as a force of magnetism. Nursing Economics Journal, 26(1), 17-25. https://pubmed.ncbi.nlm.nih.gov/18389838/

Leidel, B.A., Kirchhoff, C., Bogner, V., Stegmaier, J., Mutscher, W., \&Kanz, K., \& Braunstein, V. (2009). Is the intraosseous access route fast and efficacious compared to conventional central venous catheterization in adult patients under resuscitation in the emergency department? A prospective observational pilot study. Patient Satisfactory in Surgery 3(24), 24-31. https://doi.org/10.1186/1754$\underline{9493-3-24}$

Loon, F., Buise, M. P., Claassen, J., Dierick-van Daele, A., \& Bouwman, A. (2018). Comparison of ultrasound guidance with palpation and direct visualization for peripheral vein cannulation in adult patients: a systematic review and metaanalysis. British Journal of Anesthesia, 121(2), 358-366.

McEwen, M., \& Wills, E. M. (2018). Theoretical basis for nursing. WoltersKluwer. 
Medscape. 2019 Intravenous Cannulation. Retrieved from https://emedicine.medscape.com/article/1998177-overview

Pandurangadu, A., Tucker, J., \& Bahl, A. (2016). Patient satisfaction with nurse placed ultrasound guided peripheral IV. Emergency Medicine: Open Access, 6(325), 1-5. doi: $10.4172 / 2165-7548.1000325$

Prakash, B. (2010). Patient satisfaction. Journal of Cutaneous and Aesthetic Surgery, 3(3),151-155. https://doi.org/10.4103/0974-2077.74491

Rupp, J. D., Ferre, R. M., Boyd, J. S., Dearing, E., McNaughton, C. D., Liu, D., Jarrell, K. L., McWade, C. M., \& Self, W. H. (2016). Extravasation Risk Using Ultrasound-guided Peripheral Intravenous Catheters for Computed Tomography Contrast Administration. Academic Emergency Medicine: Official Journal of the Society for Academic Emergency Medicine, 23(8), 918-921. https://doi.org/10.1111/acem.13000

Shokoohi, H., Boniface, K., McCarthy, M., Khedir Al-tiae, T., Sattarian, M., Ding, R., Liu, Y. T., Pourmand, A., Schoenfeld, E., Scott, J., Shesser, R., \& Yadav, K. (2013). Ultrasound-guided peripheral intravenous access program is associated with a marked reduction in central venous catheter use in noncritically ill emergency department patients. Annals of Emergency Medicine, 61(2), 198-203. doi: 10.1016/j.annemergmed.2012.09.016

Sou, V, McManus C, Mifflin N, Frost SA, Ale J, Alexandrou E. (2017). A clinical pathway for the management of difficult venous access. BioMed Central Nursing, 16(64), 1-7. https://doi.org/10.1186/s12912-017-0261-z - 
Witting, M. (2011). IV access difficulty: incidence and delays in an urban emergency department. The Journal of Emergency Medicine, 42(4), 483-487. doi: https://doi.org/10.1016/j.jemermed.2011.07.030 